\title{
Finite element simulation of broadband biosonar signal propagation in the near- and far-field of an echolocating Atlantic bottlenose dolphin (Tursiops truncatus)
}

Chong Wei, Whitlow W. L. Au, Darlene R. Ketten, and Yu Zhang

Citation: The Journal of the Acoustical Society of America 143, 2611 (2018); doi: 10.1121/1.5034464

View online: https://doi.org/10.1121/1.5034464

View Table of Contents: http://asa.scitation.org/toc/jas/143/5

Published by the Acoustical Society of America

\section{Articles you may be interested in}

Estimating the sound speed of a shallow-water marine sediment from the head wave excited by a low-flying helicopter

The Journal of the Acoustical Society of America 142, 2273 (2017); 10.1121/1.5007953

Whistle description of Irrawaddy dolphins (Orcaella brevirostris) in Bay of Brunei, Sarawak, Malaysia

The Journal of the Acoustical Society of America 143, 2708 (2018); 10.1121/1.5036926

Transient response of the human ear to impulsive stimuli: A finite element analysis

The Journal of the Acoustical Society of America 143, 2768 (2018); 10.1121/1.5026240

Instantaneous Bayesian regularization applied to real-time near-field acoustic holography

The Journal of the Acoustical Society of America 142, 924 (2017); 10.1121/1.4998571

Attenuation and group speed in water-saturated granular materials at $\mathrm{MHz}$ frequencies

The Journal of the Acoustical Society of America 143, 2744 (2018); 10.1121/1.5033901

Passive acoustic detection and estimation of the number of sources using compact arrays

The Journal of the Acoustical Society of America 143, 2825 (2018); 10.1121/1.5037361 


\title{
Finite element simulation of broadband biosonar signal propagation in the near- and far-field of an echolocating Atlantic bottlenose dolphin (Tursiops truncatus)
}

\author{
Chong Wei ${ }^{a)}$ and Whitlow W. L. Au \\ Hawaii Institute of Marine Biology, University of Hawaii, 46-007 Lilipuna Road, Kaneohe, Hawaii 96744, \\ USA \\ Darlene R. Ketten \\ Department of Otology and Laryngology, Harvard Medical School, Biology Department, \\ Woods Hole Oceanographic Institution, Woods Hole, Massachusetts 02543, USA \\ Yu Zhang ${ }^{\text {b) }}$ \\ Key Laboratory of Underwater Acoustic Communication and Marine Information Technology of the Ministry \\ of Education, Xiamen University, Zengcuoan West Road, Xiamen, 361005, People's Republic of China
}

(Received 3 November 2017; revised 10 March 2018; accepted 11 April 2018; published online 2 May 2018)

Bottlenose dolphins project broadband echolocation signals for detecting and locating prey and predators, and for spatial orientation. There are many unknowns concerning the specifics of biosonar signal production and propagation in the head of dolphins and this manuscript represents an effort to address this topic. A two-dimensional finite element model was constructed using high resolution CT scan data. The model simulated the acoustic processes in the vertical plane of the biosonar signal emitted from the phonic lips and propagated into the water through the animal's head. The acoustic field on the animal's forehead and the farfield transmission beam pattern of the echolocating dolphin were determined. The simulation results and prior acoustic measurements were qualitatively extremely consistent. The role of the main structures on the sound propagation pathway such as the air sacs, melon, and connective tissue was investigated. Furthermore, an investigation of the driving force at the phonic lips for dolphins that emit broadband echolocation signals and porpoises that emit narrowband echolocation signals suggested that the driving force is different for the two types of biosonar. Finally, the results provide a visual understanding of the sound transmission in dolphin's biosonar. (C) 2018 Author(s). All article content, except where otherwise noted, is licensed under a Creative Commons Attribution (CC BY) license (http://creativecommons.org/licenses/by/4.0/). https://doi.org/10.1121/1.5034464

$[\mathrm{JJF}]$

Pages: 2611-2620

\section{INTRODUCTION}

Bottlenose dolphins have been the most studied species of all odontocete species in every area of research including biosonar research. Norris et al. (1961) demonstrated echolocation in a dolphin by covering its eyes with neoprene suction cups and detecting click signals while the bottlenose dolphin navigated its tank environment. Bottlenose dolphins emit brief (70-100 $\mu \mathrm{s})$ broadband clicks with peak frequencies as high as $120-130 \mathrm{kHz}$. The biosonar signals are emitted in a relatively narrow beam of about $10^{\circ}(\mathrm{Au}, 1993,2015)$. The anatomical structures in the acoustic pathway of biosonar production and propagation in the heads of dolphins and porpoises are extremely complex (Cranford, 1992; Cranford et al., 1996; Aroyan et al., 1992). Although there are differences in head shapes

\footnotetext{
a)Electronic mail: weichong3310@foxmail.com

${ }^{b)}$ Current address: Acoustic Research Laboratory, Tropical Marine Science Institute, National University of Singapore, 12A Kent Ridge Road, Singapore 119222, Singapore.
}

and sizes across species, the acoustic path in the odontocete's head is mainly influenced by three different structures: the air sacs and spaces; the bony structures (skull structures) which include the cranium, maxilla, and mandible; and the soft tissues which include the melon, connective tissue, musculature, blubber, etc. Complex reflection and refraction occur when the emitted biosonar signal propagates through these structures and radiates into the water. Although traditional experimental methods have been performed, the underlying mechanism of how the internal structures function in the processes is still understood only in a very general fashion.

In order to investigate the specific details of how these structures function and further increase our understanding of the mechanics of biosonar sound production and propagation, numerical modeling techniques have been applied to simulate the biosonar systems of odontocetes (Aroyan et al., 1992; Aroyan et al., 2001; Krysl et al., 2006; Cranford et al., 2014; Wei et al., 2014; Wei et al., 2016; Song et al., 2016; Wei et al., 2017; Wei et al., 2018). There is a general consensus on the roles of the skull and air sacs 
from the results of prior numerical simulation research and experimental measurements (Aroyan et al., 1992; Houser et al., 2004; Au et al., 2010; Finneran et al., 2014; Cranford et al., 2014; Wei et al., 2014; Song et al., 2016; Wei et al., 2016; Wei et al., 2017; Wei et al., 2018). Although there are differences in the geometry of the air sacs and rostrum across species, these structures act as acoustic reflectors because of impedance mismatch between them and the surrounding soft tissues directing the waves forward to form a radiating beam. The melon fills a large portion of the forehead and has a low acoustic impedance core with the impedance, gradually increasing towards the surface of the melon (Norris and Harvey, 1974). Previous studies have considered the melon as an individual structure while investigating its function in forming the biosonar beam (Jing et al., 1982; Cranford, 1988; Kloepper et al., 2012). However, evidences from the numerical simulation of acoustic propagation in the heads of dolphins by Aroyan et al. (1992) and Wei et al. (2016) have indicated that the melon is not the major entity in the dolphin's head responsible for the focusing of the biosonar beam, as suggested by Norris and Harvey (1974), Cranford (1988), and more recently by Kloepper et al. $(2012,2015)$. The numerical simulation results suggest the melon does slightly narrow the beam but it also functions as a collimator and impedance transformer. From a two-dimensional (2D) numerical simulation of sound propagating through a dolphin's head, Wei et al. (2017) showed in a pictorial fashion how echolocation signals propagated through the melon of a harbor porpoise's head in the vertical plane. They also calculated the beam pattern and sound pressure at different positions as the sound traveled through the melon. The results supported the notion that the melon functions as a collimator or acoustic waveguide in the vertical plane. However, there are other soft tissues with different acoustic properties and configurations in the animal's forehead besides the melon, such as the dense connective tissue, musculature, etc. The role of these soft tissues in forming the beam has been neglected to a certain degree, but these tissues may provide a selective advantage to dolphins. It is therefore important to determine if and how these tissues contribute to biosonar beam formation in the dolphins.

The echolocation clicks emitted by odontocetes can be roughly classified into narrowband high frequency and broadband echolocation signals (Au, 1993; Au et al., 1999). Most of the odontocetes use broadband echolocation signals, although smaller porpoises and dolphins that do not emit whistles use narrowband high frequency signals for echolocation (Au et al., 1999). There are some distinct differences in regard to the signal waveform and spectrum. Clicks from bottlenose dolphins contain 4-8 cycles within a duration of $40-70 \mu \mathrm{s}$, and have a $3 \mathrm{~dB}$ bandwidth of about $30-60 \mathrm{kHz}$ (Au, 1993; Au and Simmons, 2007). The echolocation signals used by harbor porpoises have a minimum of about 12 cycles within a duration generally greater than $100 \mu \mathrm{s}$, and a $3-\mathrm{dB}$ bandwidth of about $10-15 \mathrm{kHz}$ (Au et al., 1999). Although these two types of echolocation signals have different characteristics, the animals seem to have a similar physical mechanism to excite vibration of the right phonic lips to produce projected signals (Madsen et al., 2013). It is still unknown whether the anatomical difference of the forehead is the reason for the differences in the projected signals.

In this study, a high-resolution computer tomography (CT) scan was used to create an impedance model of an Atlantic bottlenose dolphin's (Tursiops truncatus) head. A finite element model was then used to simulate the acoustic propagation of an echolocation click emitted from right phonic lips into the water after propagating through the complex structures in the animal's head in the vertical plane. The acoustic field and the output signals on the animal's forehead, as well as the farfield transmission beam pattern of the echolocating dolphin, were calculated and compared with previous measurement results. The comparison results showed the similarity between the simulation results and the measured results. The role of the main structures on the sound propagation pathway such as the air sacs, melon, and connective tissue was investigated.

\section{METHODS}

\section{A. Acoustic impedance model construction}

A CT scan was used to extract the anatomical geometry of the dolphin's head. The specimen involved in this study was an expired stranded animal. The Institutional Animal Care and Use Committee (IACUC) Animal Use Committee of the Woods Hole Oceanographic Institution (WHOI) has reviewed and granted a blanket IACUC approval for handling and examining the cadaveric specimens. The CT of the head of a bottlenose dolphin was provided by the WHOI Biology Department. The specimen was a ten-yearold male bottlenose dolphin. The head was obtained from the carcass of the animal after it had died and was immediately frozen to avoid decomposition artifacts. A Siemens Volume Zoom CT scanner was used to scan the head. A spiral protocol was employed with $120 \mathrm{kV} \times 125 \mathrm{~mA}$ and $1 \mathrm{~mm}$ acquisitions. Figure 1(a) shows one of the slices from the CT scan in the sagittal plane. No tissue property measurements were performed on the bottlenose dolphin's head. However, the studies regarding the relationships between animal tissue properties and Hounsfield Unit (HU) values have shown similar results from different species (Soldevilla et al., 2005; Wei et al., 2015). Therefore, we assumed that the relationships between tissue properties and $\mathrm{HU}$ values in the bottlenose dolphins are similar to those of the Yangtze finless porpoise (Neophocaena asiaeorientalis) obtained by tissue property measurements in Wei et al. (2015). With the distribution of HU values derived from the CT data of bottlenose dolphin, we used the relationships obtained by Wei et al. (2015) to calculate the distribution of acoustic impedance in the head of the bottlenose dolphin in this study. The same approach was applied in the study of Wei et al. (2017) to configure the acoustic impedance model of the head of a harbor porpoise. In this paper, Software Mimics 10.1 (Materialise, Belgium) was used for CT data analysis, a distribution of HU values of each structure in the head of the bottlenose dolphin 

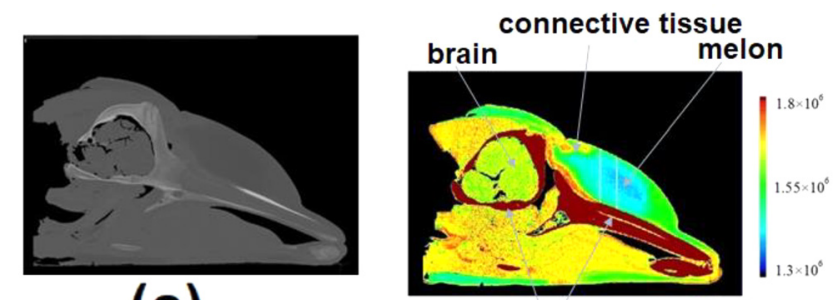

(a)

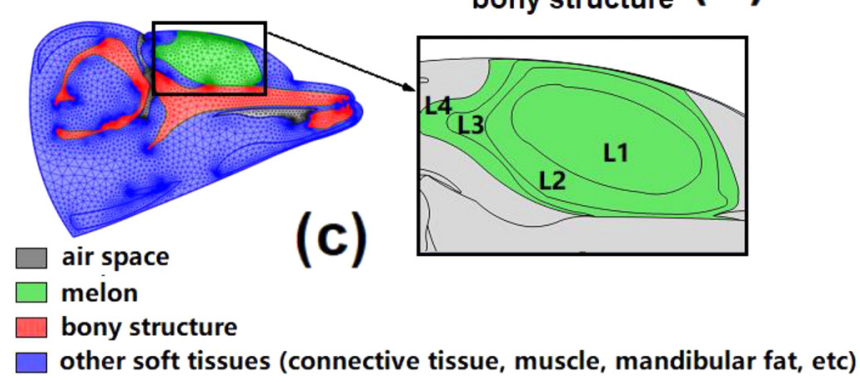

FIG. 1. (Color online) (a) One of the midline, sagittal slices from the CT scan of the head of an Atlantic bottlenose dolphin. The gray level in the figure represents the different HU values; (b) a 2D acoustic impedance model of the head of the Atlantic bottlenose dolphin based on the sagittal slice through the right phonic lips. (c) The impedance model overlaid with a triangular mesh grid used in the later simulation, the model includes the main structures in the dolphin's head: air spaces, bony structure, melon, connective tissue, blubber, mandibular fat, brain, musculature, etc. For display purposes, the element size is enlarged by a factor of 8 over the element size used in the simulation. $L 1, L 2, L 3$, and $L 4$ represent four distinctive contours in the melon, the acoustic impedance values of the points in the melon area increase from $L 1$ to $L 4$.

sample was exported. The CT data were converted into an acoustic impedance model of the head of the animal using prior knowledge of HU-to-sound velocity and HU-to-density relationships in the heads of odontocetes (Wei et al., 2015), which are shown in Fig. 1(b). The average values of sound velocity and density we adopted for the various structures in the dolphin's head are shown in Table I. From the CT data, the distributions of sound velocity and density of the melon of the bottlenose dolphin showed significant inhomogeneities, both sound velocity and density values increased from the inner core to the outer layer, which was consistent with the earlier findings by Norris and Harvey (1974). Although the grid points were separated by at least $1 / 10$ of a wavelength at the center frequency, the peaks in the distribution of acoustic impedance mapped out into four distinctive contours [see Fig. 1(c)]. The "layers" were composed by many discrete points based on the CT data in a

TABLE I. The average values of sound velocity and density of various structures in the dolphin's head used in the simulation.

\begin{tabular}{lcc}
\hline \hline Structures & Sound velocity $(\mathrm{m} / \mathrm{s})$ & Density $\left(\mathrm{kg} / \mathrm{m}^{3}\right)$ \\
\hline Blubber & 1473 & 990 \\
Muscle & 1590 & 1031 \\
Mandibular fat & 1431 & 970 \\
Melon & $1344-1480$ & $934-982$ \\
Connective tissue & 1553 & 1006 \\
Bony structure & 3800 & 2000 \\
\hline \hline
\end{tabular}

similar fashion as Aroyan et al. (1992) and Cranford et al. (2014).

For the live animals, the volume of the air in the air space is dynamic. There have been little to no empirical measures of air sacs volumes in live animals to suggest how much air volume can be contained within the air space at any given moment during echolocation, nor what the ultimate deformations of the air space might be. Thus, the shape of the air sacs during echolocation can only be approximated according to the CT scan data and the anatomic information provided by the previous studies (Cranford et al., 1996; Houser et al., 2004). The air sacs of our specimen were partially inflated during the CT scanning, therefore, we assumed that the nasal passage, vestibular sac, and premaxillary sac are partially inflated in this model. The beam patterns in these air situations are therefore estimated. The impact from the different volumes within the air space will be investigated in future work.

\section{B. Modeling}

A finite element model (FEM) was used to simulate the processes of biosonar signal emission and propagation through the tissues of an Atlantic bottlenose dolphin's head and into the surrounding water. A 2D geometrical model of the animal's head that passed through the right phonic lips was exported from the sagittal cross section of CT data (54 cm length $\times 32 \mathrm{~cm}$ height). In the coronal view of the CT data, we observed that the length of the right phonic lips was approximately $22.5 \mathrm{~mm}$ and oriented nearly perpendicular to the midline of the animal's head, the distance between them are approximately $6.5 \mathrm{~mm}$. We selected the slice which was closest to the midline to configure the 2D numerical model. The model included two main parts: the head of the dolphin and the surrounding sea water. All of the structures, such as the melon, blubber, brain, musculature, mandibular fat, connective tissue, bony structure, vestibular sac, nasal passage, and premaxillary sac were included in the head. COMSOL Multiphysics finite element modeling software (Stockholm, Sweden) was used to conduct the simulation and analyze the data. Second-order Lagrangian elements are commonly used in COMSOL Multiphysics to discretize the geometry and the solution for solving most physics problems, including acoustics. The second-order element can more accurately represent the curved boundaries of elements and represents a good compromise between accuracy and computational requirements. Besides, the triangular elements have better performance in dealing with irregular boundaries and complex structures such as the oddly shaped animal head. Therefore, the second order triangular mesh grid was selected to overlay the whole model. The mesh layout in the lateral view of the model is displayed in Fig. 1(c). A mesh refinement analysis was performed to choose the optimal element size for this model. For obtaining the solution with sufficient numeric precision, we set the maximum element size as ten elements per wavelength of the center frequency of the excitation signal at the source in the simulation $\left(\lambda=c_{\text {water }} / f_{c}\right)$. The low-reflecting boundary condition 
(Bérenger, 1994) was applied to model the signals propagation through the free space and into the seawater.

The finite element computation was operated in the time domain based on the physics of sound propagation in a fluid. The inhomogeneous acoustic wave equation describing the transient acoustic phenomena in a stationary fluid was solved at each grid. The equation can be written as

$$
\frac{1}{\rho c_{s}^{2}} \frac{\partial^{2} p}{\partial t^{2}}+\nabla \cdot\left(-\frac{1}{\rho} \nabla p\right)=Q_{m},
$$

where $\rho$ denotes the equilibrium density $\left(\mathrm{kg} / \mathrm{m}^{3}\right), c_{s}$ is the sound velocity $(\mathrm{m} / \mathrm{s}), p=p(x, t)$ is the acoustic pressure (Pa), and $Q_{\mathrm{m}}$ is a monopole source (Wei et al., 2016). The density and the sound velocity can both be non-constant in space. They are assumed to vary with time on scales much larger than the period of the acoustic waves and are therefore considered time independent in Eq. (1).

There are two sets of phonic lips located on both the left and right sides of the membranous nasal septum below the blowhole (Cranford et al., 1996). The phonic lips are surrounded by a number of air sacs, the sound generation complex associated with the phonic lips is supported by a stiff cartilaginous blade and is anchored by a stout ligament (Cranford et al., 2000). Previous studies by Cranford and his colleagues (Cranford et al., 2000) operated high-speed video endoscopy and acoustic monitoring to observe movements in both sets of phonic lips during sound production. They showed that the phonic lips open and close immediately after air is forced through them, which caused the surrounding tissues to vibrate. A short-duration impulsive sound with subsequent damped oscillations is generated at this moment. In this study, a short-duration broadband pulse from the right set of phonic lips was used since acoustic measurements by Madsen et al. $(2010,2013)$ indicated that only the right phonic lip is involved in the production of biosonar clicks. In the sagittal slice in Fig. 2(b), the size of the right phonic lips is approximately $3-4.5 \mathrm{~mm}$, which is significantly smaller than a wavelength $(1.85 \mathrm{~cm})$ used in the simulation. Thus, a point source was used to model the source region in this study. The pulse generated by the source can be written as

$$
Q_{m}=-A e^{-2 \pi^{2} f_{0}^{2}\left(t-t_{p}\right)^{2}} \sin \left(2 \pi f_{0} t\right)
$$

where $A$ is the pulse amplitude $(\mathrm{Pa}), f_{0}$ is the center frequency $(\mathrm{Hz}), t_{p}$ is the time from the onset of the signal to its
D

C

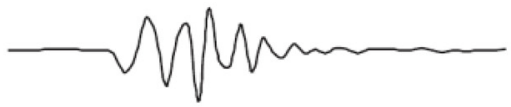

B

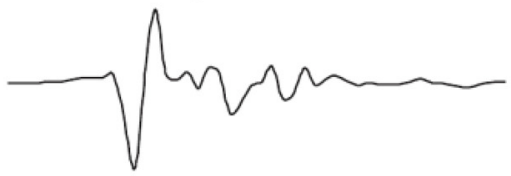

A

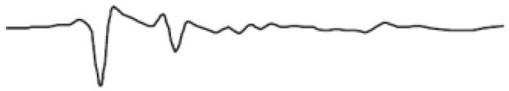

E

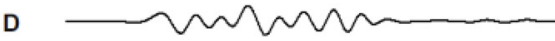

C

B

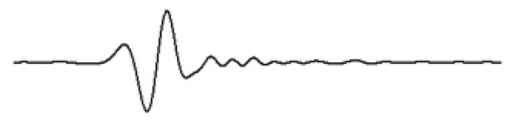

A

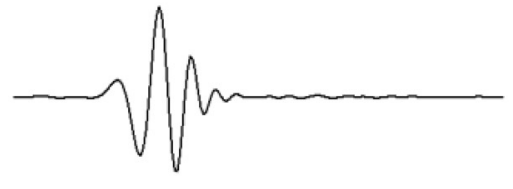

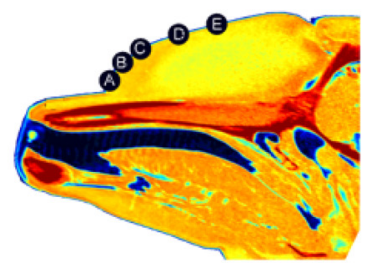

Results from Au et al. (2010)
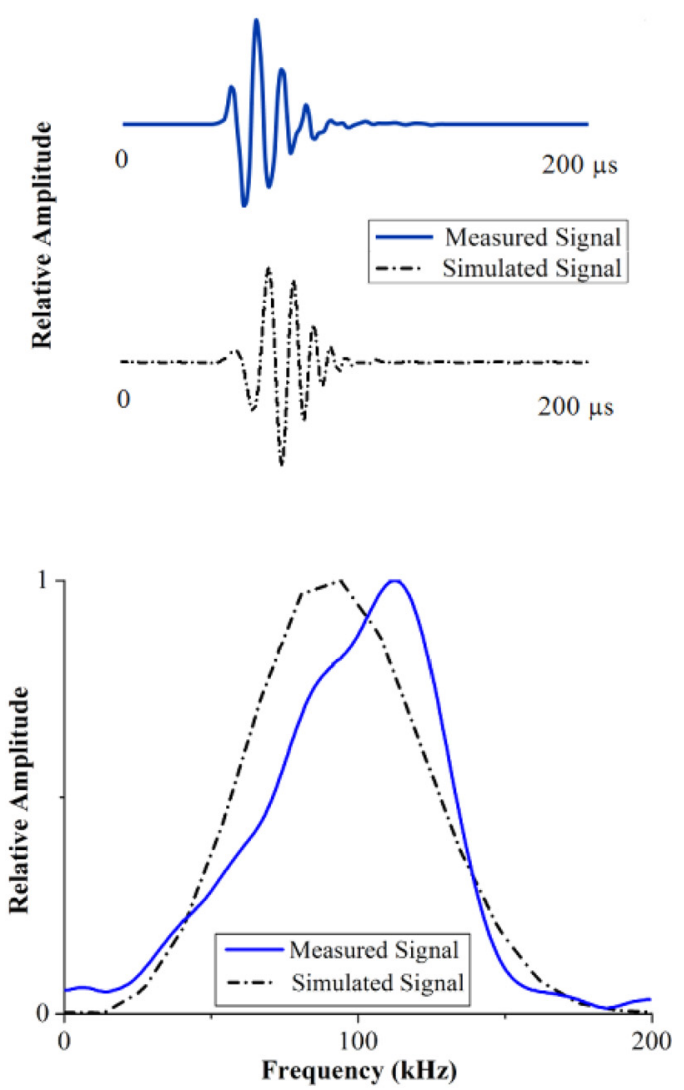

Results from this study

(a)

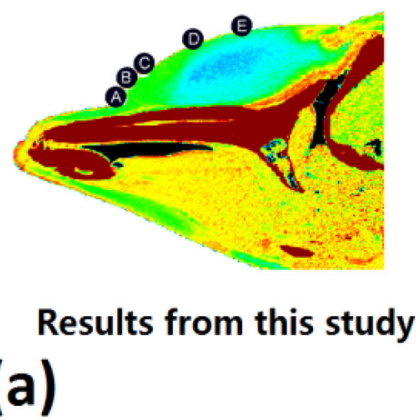

(b)

FIG. 2. (Color online) Comparison of the simulation results to biosonar measurements. (a) Simulation results and measured results on the surface of the echolocating bottlenose dolphin's head. Points A-E represent the five receiving points located at the same positions of suction cup hydrophones in the study by Au et al. (2010). The amplitudes of the received signals from the simulation results in (a) were relative to the highest amplitude at point A. (b) Signal waveform (above) and frequency spectrum (below) both measured (in the farfield) and from the finite element calculations (at $0.6 \mathrm{~m}$ from the sound source on the main beam axis). The dashed lines represent the simulated results from this study and the solid lines represent the measurements from an echolocating dolphin. 
peak amplitude (s), and $t$ is the time (s). The time of the pulse had to satisfy the following equation:

$$
t_{p}-\frac{1}{f_{0}}<t<t_{p}+\frac{1}{f_{0}} .
$$

\section{RESULTS}

A comparison of the results of the finite element (FE) simulation to actual acoustic measurements by previous work (Au et al., 2010; Au, 1993) is shown in Figs. 2 and 3. Au et al. (2010) measured the acoustic field on the forehead of an echolocating Atlantic bottlenose dolphin by placing a linear array of suction cup hydrophones along the midline of the animal's forehead to record the echolocation signals. In this study, the acoustic field on the animal's forehead at five receiving points (from $\mathrm{A}$ to $\mathrm{E}$ ), which corresponded approximately to the same positions as the suction cup hydrophones in the study by Au et al. (2010) were determined. The comparison results are shown in Fig. 2(a). It should be noted that with a live animal, the situation is dynamic and there are slight variations in both waveform and amplitude from click to click.

In the FE simulation results, all the peak-to-peak amplitudes of the output signals were normalized according to the highest peak-to-peak amplitude to obtain relative amplitudes. From the transient solution, very detailed sound propagation processes inside the forehead could be observed when the time steps were set as $0.8 \mu$ s. Complex reflections and refractions occurred in the animal's head when the exponentially damped sinusoid traveled through the oddly shaped internal structures with inhomogeneous impedance distributions. Indirect and direct signal components combined to form the received signals with different characteristics at each receiving point on the surface of the forehead. Au et al.

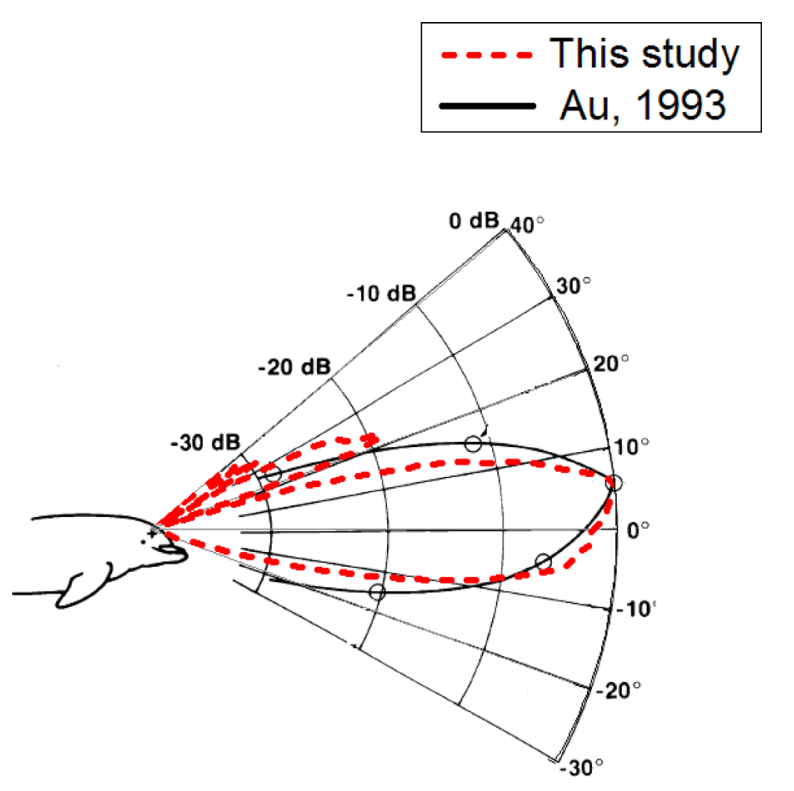

FIG. 3. (Color online) Comparison between the beam pattern calculations from the simulation results and actual measurements by Au (1993) in the vertical plane. The solid line represents the measurement results from $\mathrm{Au}$ (1993) and the dashed line represents the simulated data in this study.
(2010) determined that the major axis of the radiation beam was located at the anterior portion of the forehead and very little energy was radiated from the top of the forehead in the vertical plane. From the signal amplitude distribution in Fig. 2(a), the relative amplitudes of points A, B, and C are higher than those of points D and E. The signal amplitudes drop off when the positions of the receiving points move to the top of the head, indicating the major axis of the beam is in the region between point $\mathrm{A}$ and point $\mathrm{C}$. The simulation results are qualitatively consistent with the conclusions of $\mathrm{Au}$ et al. (2010).

The waveform and its corresponding spectrum are shown in Fig. 2(b). The location of the received signal was set at $0.6 \mathrm{~m}$ from the sound source on the main beam axis. According to Finneran et al. (2016), the nearfield/farfield transition distance of bottlenose dolphin is $\sim 0.4 \mathrm{~m}$ from the blowhole. Therefore, the signal in Fig. 2(b) is considered as the transmitted signal in the farfield. The waveform of the simulated signal and measured signal both contain four cycles, and the durations of the signals are approximately $40-50 \mu \mathrm{s}$. The peak frequency of simulated signal is close to $100 \mathrm{kHz}$, approximately $10 \mathrm{kHz}$ lower than that of the measured signal. The 3-dB bandwidths of both signals' corresponding spectrum are approximately $50 \mathrm{kHz}$ and $45 \mathrm{kHz}$, respectively. The comparison in Fig. 2 indicates the similarity between the simulation results and the measured results, indicating the signal in Fig. 2 is a typical broadband echolocation signal of an Atlantic bottlenose dolphin.

The vertical beam pattern in the farfield of the FE simulation was plotted by determining the peak-to-peak sound pressure of an echolocation click spreading from the source over a circle with a radius of $1.2 \mathrm{~m}$. The results are compared with the acoustic measurements in the farfield performed by Au (1993), as shown in Fig. 3. The elevation of the simulated beam was $5.3^{\circ}$, very close to the results of previous measurements (Au et al., 1986; Au, 1993). The 3-dB beamwidth calculated from the FE simulation was $11.1^{\circ}$, slightly greater than the measured data $\left(10.2^{\circ}\right)$ from $\mathrm{Au}(1993)$.

The boundaries of the connective tissue can be clearly distinguished in the CT scan data and impedance model since there is a significant increase in the acoustic impedance compared to the surrounding tissues, such as the melon. The connective tissue envelops the posterior portion of the melon and forms the supporting elements of the animal's phonic lips (Cranford et al., 1996). In order to investigate the role of the connective tissue in the processes of sound production and propagation, and compare it to the other main structures such as the air sacs and melon, three hypothetical cases were simulated and compared with the "full head" case. We used the same method for plotting Fig. 3 to obtain the beam pattern results for the three hypothetical cases and compared with the results of "full head" case [Fig. 4(c) is the same as the dashed line in Fig. 3]. In the "full head" case, all of the structures in the animal's head were included. In case I, only the air components associated with the air sacs were removed from the "full head" (replaced with voxel values similar to that of "neighboring" soft tissues) model and the rest of the structures remained the same as those in the "full head" so that no air sacs were present in the animal's head 
(no vestibular sac, nasal passage, premaxillary sac, etc.). In case II, only the connective tissue was removed from the "full head" (replaced with voxel values similar to that of "neighboring" soft tissues) and the rest of the structures remained the same as those in the "full head" so that there was no connective tissue embracing the melon. In case III, only the impedance gradient of the melon was removed from the "full head" case (the acoustic properties of all layers in the melon were set the same as that of the outer layer of the melon) and the rest of the structures remained the same as those in the "full head" so that the melon was homogeneous. Case IV represented the "full head." The comparison of the beam patterns and the acoustic field results are shown in Fig. 4 and Fig. 5, respectively.

From the comparison results in Fig. 4, more side lobes were found in the three hypothetical cases, especially in case I. The polar plot of case I shows that part of the energy of the biosonar beam propagates to the top and bottom in the absence of the air sacs. The large acoustic impedance mismatch between the air components and surrounding tissues causes the air sacs to act as acoustic reflectors directing the beam forward and reducing side lobes. When the connective tissue was removed in case II, the side lobes were increased and the elevation of the main beam decreased more than $10^{\circ}$.

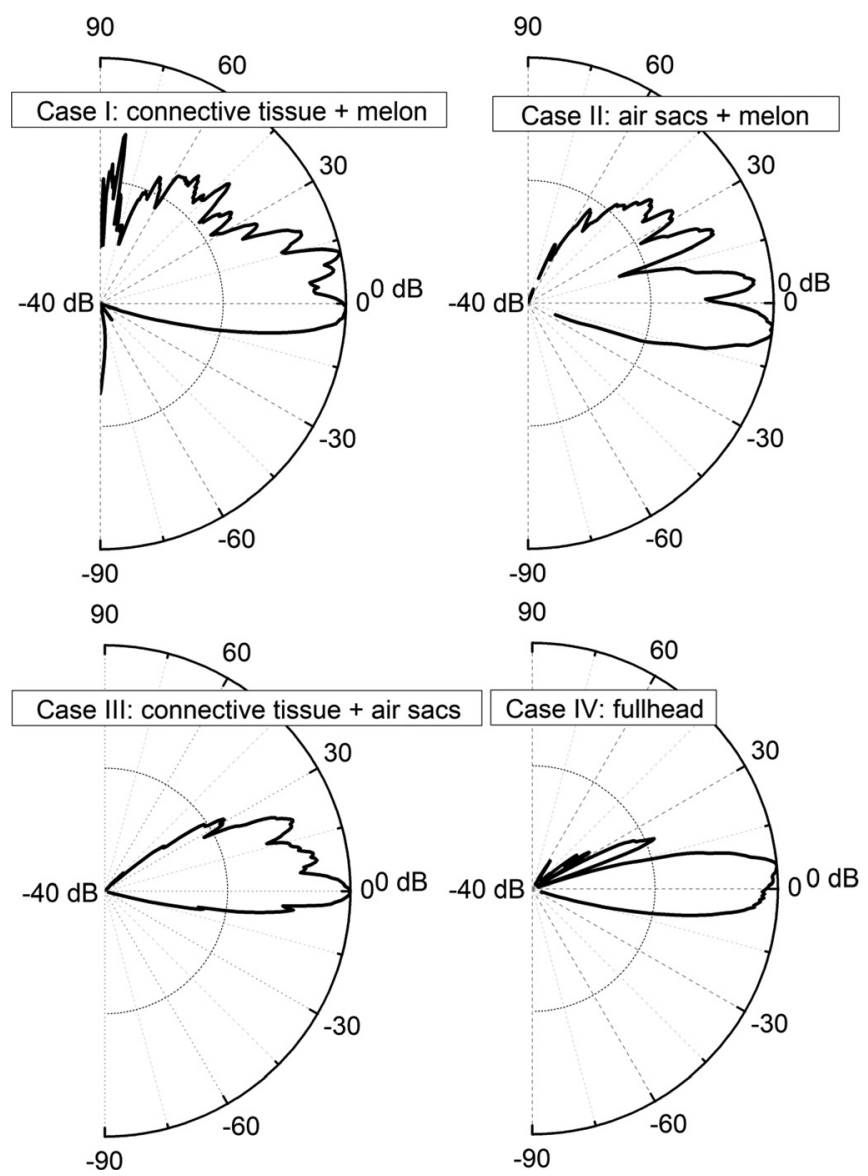

FIG. 4. The modeled, farfield vertical beam patterns for four different model manipulations. (a) Case I: full head with only the air sacs removed; (b) case II: full head with only the connective tissue removed; (c) case III: full head with a homogenous melon; (d) case IV: full head including the skull, melon, air sacs, connective tissue, blubber, musculature, mandibular fat, etc. (d) was plotted by using the data represented by the dashed line in Fig. 3.
In case III, when the inhomogeneous melon was replaced by a homogeneous melon, the side lobes were greater than those in full head case (but significantly less than those in case I and case II), and the elevation angle of the main beam was found to be approximately $5^{\circ}$ lower compared to the full head case.

In order to further examine the role of the air sacs, connective tissue, and melon in the propagation of echolocation clicks through the forehead and into the water, a time history of the click wavefront traveling through the forehead was determined for each case. The wavefront at five moments (T1-T5) was captured from the analysis and shown in Fig. 5 to visualize the specific changes in the wavefront as the click propagated through five positions in the forehead.

When the click was emitted from the source point (T1), the wavefronts in cases II-IV were approximately the same, indicating the connective tissue and melon barely impacted the waves. However, the waves in case I were distinctively different from those in the other three cases where there were no air sacs acting as acoustic reflectors to direct the click forward. As a result, a more circular wavefront was formed. When the click arrived at the melon (T2) in case I, some of the energy propagated into the water from the top of the head and some of the waves were reflected by the cranium. The wavefront in the other three cases at this moment were very similar. Only a slight difference in case II can be observed: the beam was slightly wider in case II when the connective tissue was removed compared to those in case III and case IV. This difference was more distinct after the waves entered the melon (T3); the beam in case II was significantly wider than those in case III and case IV. The connective tissue constrains most of the energy to within the melon region as the wave travels through it. As time went from T3-T5, the inhomogeneous melon (cases I, II, and IV) with its impedance gradient caused the wavefront to bend as it traveled through the melon region. The angle of outgoing beam was changed by the melon (compared case III to cases I, II, IV). The comparison results indicated the collimation or acoustic waveguide role of the melon.

\section{DISCUSSION}

Previous studies (Au et al., 1999; Au, 1993) have shown that the size of the animal's head has a certain relationship with the outgoing beam properties. However, very little is known about whether the anatomical differences will also cause the different characteristics of the outgoing signals across species. We previously simulated sound propagation in the heads of narrowband echolocating porpoises (Wei et al., 2017; Wei et al., 2018) by applying an exponentially damped sinusoid to represent the driving force at source excitation, shown as the following equation:

$$
Q_{m}=A e^{\gamma t} \sin 2 \pi f_{0} t
$$

The bandwidth of Eq. (4) was significantly narrower compared to the broadband signal in Eq. (2) used in this study (see Fig. 6). In the beginning, we assumed the different characteristics of the echolocation clicks could be attributed to anatomical differences between dolphins and porpoises, 
T1
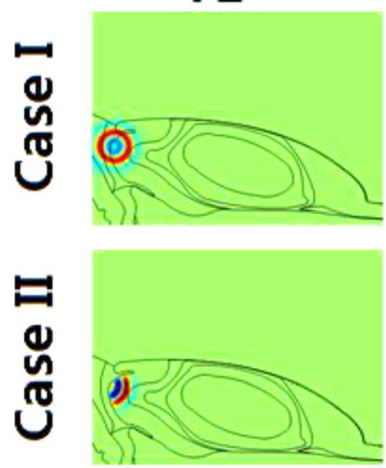

目

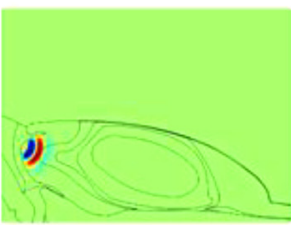

Z

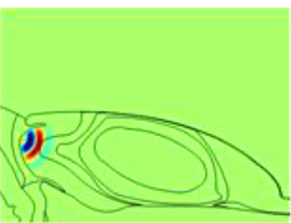

T2
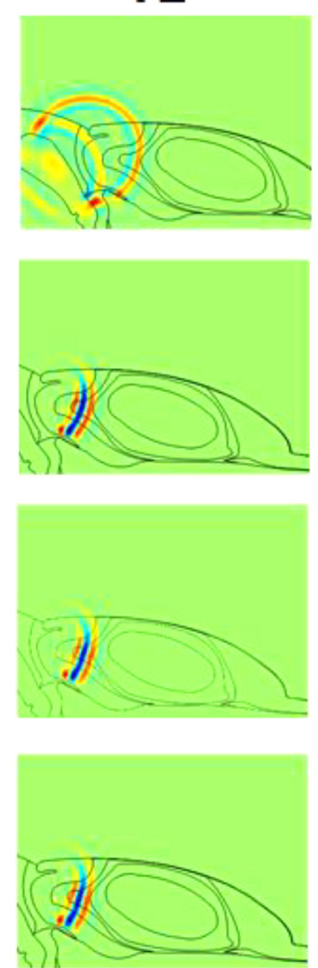

T3
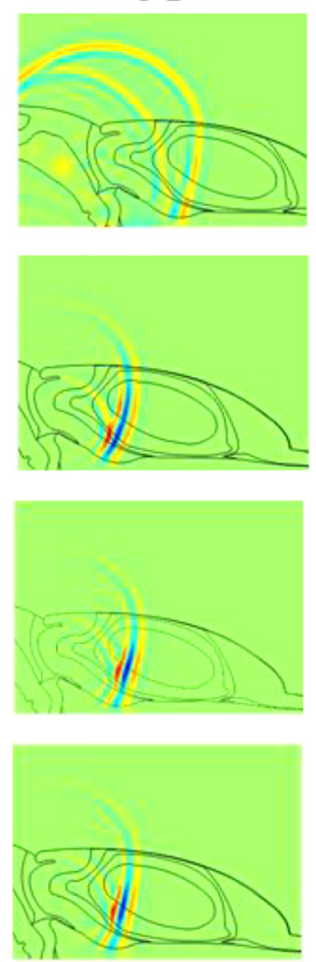

T4
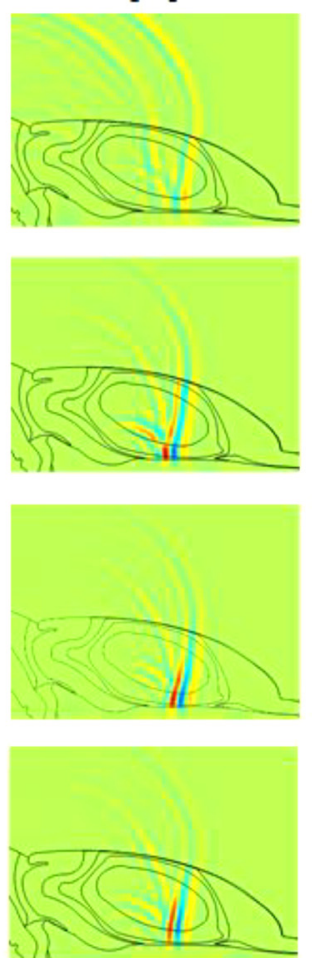

T5
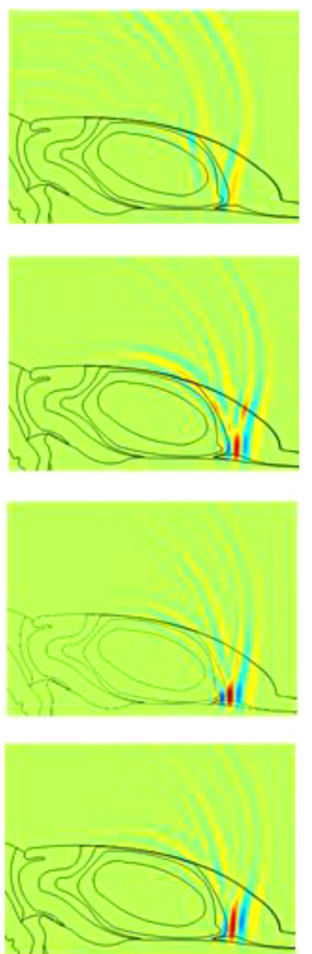

FIG. 5. (Color online) The time history of the click wavefront traveling through the animal's forehead in each case study. $T 1$ represents the moment a click was emitted from the source excitation, $T 2$ represents the moment the click enters in to the melon, $T 3$ represents the moment the click travels through onethird of the melon, T4 represents the moment the click travels through two-thirds of the melon, and T5 represents the moment the click leaves the melon. The color intensity corresponds to the amplitude of the propagating wave.

such as different head shape and size, length of the rostrum, size of the melon, shape of the connective tissue, etc. In order to examine our hypothesis, all the settings and parameters in this bottlenose dolphin model were held constant, except we used Eq. (4) as the driving force at the right phonic lips. The calculated outgoing signals and beam patterns did not resemble measured data even though we tried to use different parameters in Eq. (4) (the values of the frequency, damping rate, etc.). There were more cycles (at least ten cycles) contained in the waveforms of the received signals and the bandwidth of the signal received in the farfield was narrower (approximately $25-35 \mathrm{kHz}$ ). The signals looked more similar to the narrowband high frequency signals. In another simulation, the short-duration broadband pulse [Eq. (2)] was used as the driving force in a Phoceona model. The results obtained did not match the corresponding measurement results. However, broadband signals were produced when the broadband pulse was used as the driving force. Therefore, switching the driving waveform at the phonic lips for the two models suggested that the driving force may be responsible for the two types of biosonar signals typically measured for dolphins and porpoises and not the anatomical differences between the two species. Further research to reveal the reasons for using the different driving forces in sound sources of dolphins and porpoises is needed. One possible explanation to explore is the ecology of the animals. Broadband signals provide more acoustic information to the animals, but a porpoise might also change its signals by using a different driving force to avoid being heard by predators, such as killer whales. Thus, the information returning in echoes might not be as information-rich, but the risk of predation would be lower.

Au et al. (2010) suggested the air sacs play a big role in the acoustic signals being projected toward the front of the animals rather than toward the top of the animal's forehead. The simulation in this study provides the details of a sound transmission pathway inside the head by showing a broadband signal travels through the animal's biological structures frame by frame. The simulation results (see Fig. 5) showed that a directional beam is formed by reflections off the air sacs before entering into the melon, strongly agreeing with the measurement results from Au et al. (2010). Wei et al. (2017) modeled the sound propagation in the head of a harbor porpoise and suggested that the melon function as a collimation of the outgoing biosonar signal. A similar role of the melon in this bottlenose dolphin's head can be found in this study by comparing the results of case III with case IV in Fig. 5. When the gradient in the acoustic impedance is removed from the melon, the waves propagate directly to the surface of the rostrum after being reflected by the air sacs (see case III in Fig. 5). When the inhomogeneous melon is present (see case IV in Fig. 5), the melon guides the waves along the low-impedance core, which is positioned almost in the middle of the melon. The direction of the beam is changed slightly when the waves travel through the region with the gradient in the acoustic impedance. Although there are anatomical differences in the forehead structures between the Tursiops and Phoceona, the melon of both 

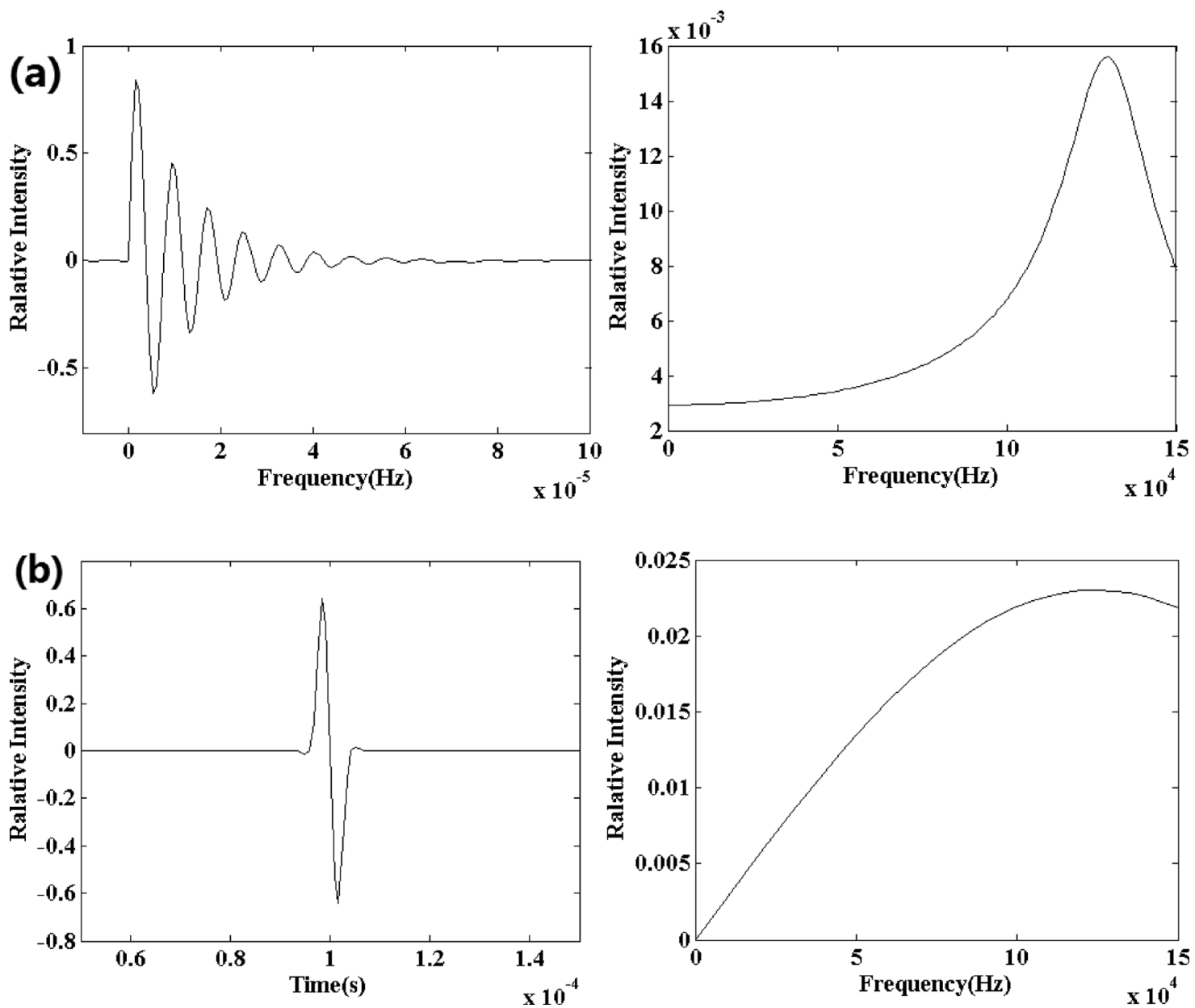

FIG. 6. Comparison of the narrowband exponentially damped sinusoid (a) used by Wei et al. (2017) and Wei et al. (2018) and the short-duration broadband pulse (b) used in this model.

species functions as an acoustic waveguide to collimate the projected biosonar signal.

The melon, as an adipose tissue with a sound speed gradient has been mistakenly attributed as a focusing lens for biosonar signals (Norris and Harvey, 1974; Cranford, 1988; Kloepper et al., 2012; Kloepper et al., 2015). The measurements by Finneran et al. (2016) clearly showed that focusing does not occur as hypothesized by Kloepper et al. (2012, 2015 ) and the results of our numerical simulation are consistent with the measurement results of Finneran et al. (2016). Some beam width narrowing does occur but the major structures in the formation of the beam are the skull and air sacs (Aroyan et al., 1992; Wei et al., 2016; Wei et al., 2017) because huge impedance mismatches exist between the solid component (skull), air component (air sacs), and the fluid components (the soft tissues in the forehead and the water outside the head). The dense connective tissue, which is connected to the main body of the melon, has a role in that it is required for muscular action that might act on the melon (Cranford et al., 1996; McKenna, 2005; Harper et al., 2008). The results showed that the connective tissue prevents the waves from radiating into the water from the top of the forehead and transfers most of the energy into the melon because it has a significantly higher acoustic impedance compared to the melon. Overall, it acts as a transition region between the sound source and melon to ensure the waves enter into the melon effectively after reflecting off the air sacs. It should be noted that the features and anatomic configurations of the connective tissue in the heads of different odontocete species are different (Cranford et al., 1996) and the different anatomical arrangements could result in varying the propagation characteristics through the connective tissue across species.

A prior study by Cranford et al. (2014), who used a vibroacoustic finite element model to simulate acoustic beam formation in the bottlenose dolphin, suggested that the biosonar beam is focused in a series of stages. The structures involved in the different stages were defined as the skull, melon, nasal air spaces, and the configuration details of the sound generation apparatus. Our results showed some agreement with this by providing a more detailed sound propagation process and displaying how omnidirectional waves emitted from the source can form a highly directional beam by the combined effects of acoustic reflections and refractions from the internal anatomic structures. However, in this study the collimation role of the melon was emphasized. There are some significant differences between both studies. First of all, in order to closely represent an echolocating dolphin, a broadband transient signal was applied to describe the driving force at phonic lips to simulate the sound production and propagation in dolphin's head. The transient solution results obtained 
from this study proved that the output of the model would be dramatically changed if different signals were used for the driving force. There are no statements regarding the signal at the driving source found in the study of Cranford et al. (2014). Second, for the purpose of controlling the computational overhead in the three-dimensional (3D) model, Cranford et al. (2014) placed a hemispherical screen at $36 \mathrm{~cm}$ from the presumed sound source to map the sound pressure level. Thus, the results of Cranford et al. (2014) are based on the biosonar beam formation in the nearfield or close to the nearfield/farfield transition. Using a $2 \mathrm{D}$ versus a $3 \mathrm{D}$ model has the advantage of decreasing computational time, decreasing computational resources, and improving accuracy because the sizes of the elements in the model can be much smaller and provide greater spatial resolution. It allows us to obtain important qualitative information on the transition from the acoustic nearfield to farfield $(1.2 \mathrm{~m})$. Third, the results obtained from the model in this study were compared to the signal characteristics and beam properties of actual bottlenose dolphins. Our numerical results showed good correspondence to the acoustic measurements. Cranford et al. (2014) only compared the simulated beam pattern to the measured one. The results of the beam pattern could change significantly when the different driving signals with different characteristics are set (function, bandwidth, etc.). Finding the similarity of signal characteristics and beam properties simultaneously between the finite element modeling and measurement results is one of the highlights in this study. Finally, we provided insight into the important role that the connective tissue plays as sound was transferring to the melon and discussed the impact to the characteristics of outgoing signals when different driving signals at the source excitation were used.

The previous study (Moore et al., 2008) demonstrated that dolphins can control the width of the echolocation beam in target detection. Based on the vertical model in this study, there are several potential factors that could contribute to variation in the outgoing beam. Animals could change the frequency of the driving signal at the source to vary the beamwidth. Moreover, animals could change the volume of the air within the premaxillary and vestibular sacs associated with the nasal passages near the sound source to alter the shape of the reflect interface, causing the biosonar signals to reflect to different angle when they are emitted from the phonic lips. Muscular deformation of the forehead could also be another important factor to help animals control the outgoing beam. In the study of Moore et al. (2008), they mentioned that "slight deformations of the melon were observed as a melon 'squeeze', suggesting that subtle variations in melon geometry/lipid density were potentially performed by the dolphin." As the shape of the melon changes, it would also alter the shapes of other soft tissues connected to the melon in the forehead, such as the connective tissue which embraces the melon. Furthermore, it could also change the position of the phonic lips to project the signals. Therefore, beam steering is achieved by cooperation of the entire structures in the animal's forehead. A study of Zhang et al. (2017) manipulated a finless porpoise's forehead in a finite element model by changing the size of the melon, changing the length and orientation of the vestibular sac, and compressing the head to show that the outgoing beam can be changed. Although these deformations in this study (Zhang et al., 2017) might not occur in the heads of the actual animals, it still provides general information about how the beam might be manipulated.

It should be noted that the wave radiation from a point source in a 2D model and 3D model are different but only slightly in this study. In 2D simulations, waves from a point source spread circularly but not spherically due to the dimension difference, essentially similar to a slice through a 3D cylindrical wave. The spherical loss in 3D follows a $1 / r$ pattern, the waves in 2D drop off in amplitude less rapidly then spherical waves in 3D. However, the spreading losses would be minimal since the distance of sound propagation in this study is small. Our approach is similar to the approach that has been used by ocean acousticians for over one-half century in computing long range propagation. A 2D slice is taken from a 3D ocean and the wave equation is solved by a variety of techniques such as normal mode, parabolic simulation, etc. These techniques have been validated over time by propagation measurements. Our simulation has also been validated by the consistent nature of our results compared with empirical measurements. Apparently, the waves tend to remain within the $2 \mathrm{D}$ plane with very little measurable contributions from any 3D effects. The model in this study only represents a $2 \mathrm{D}$ approximation of the beam pattern in the vertical plane. The results would be improved if the modelling could be extended to a 3D model in future work when more computational power is available to us. However, it is important to realize that our $2 \mathrm{D}$ approximation provided very consistent results with measurement results and therefore lends credence to our approach.

\section{CONCLUSIONS}

A 2D finite element model was constructed based on a high-resolution CT scan and was used to simulate the process of a short-duration, broadband impulsive sound emitted from the right phonic lips into the water after propagating through the complex anatomic structures in a bottlenose dolphin's head in the vertical plane. The waveforms of the transmitted signals on the forehead and in the farfield, as well as the properties of the beam pattern in the farfield, were compared with actual biosonar signal measurements on and around the head of the live animals. The simulation results were consistent with the acoustic measurement results. The detailed sound propagation process was calculated and the time history of the click wavefront traveling through the forehead at five different moments was captured and compared. The comparison results suggested that the air sacs are the major contributor in forming a directional beam at the beginning when the sound was emitted from the source. The connective tissue plays an important role of effectively transferring the sound to the melon and preventing the sound from escaping from the top of the forehead. The melon functions as a collimator or acoustic waveguide directing the waves along the low-impedance core. Finally, the study examined the driving signal at the source of 
echolocation in bottlenose dolphins (broadband clicks) and harbor porpoises (narrowband clicks). The results indicated that the signal waveform of the driving force is more likely responsible for the two different types of projected signals and not the differences in the anatomy of the forehead.

\section{ACKNOWLEDGMENTS}

This work was financially supported in part by the National Science Foundation of China (Grant Nos. 41276040, 11174240, 31170501, and 31070347), the Natural Science Foundation of Fujian Province of China (Grant No. 2012J06010), Ministry of Science and Technology of China (Grant No. 2011BAG07B05-3) and State Oceanic Administration of China (Grant No. 201105011-3). The Project was sponsored by the Scientific Research Foundation for the Returned Overseas Chinese Scholars, State Education Ministry. This is HIMB contribution No. 1630 and SOEST contribution No. 9452. One of the authors, C.W., was supported by the China Scholarship Council. The funders had no role in study design, data collection and analysis, decision to publish, or preparation of the manuscript. The role of the Office of Naval Research in supporting W.W.L.A. is also acknowledged.

Aroyan, J. L. (2001). "Three-dimensional modeling of hearing in Delphinus delphis," J. Acoust. Soc. Am. 110(6), 3305-3318.

Aroyan, J. L., Cranford, T. W., Kent, J., and Norris, K. S. (1992). "Computer modeling of acoustic beam formation in Delphinus delphis," J. Acoust. Soc. Am. 92(5), 2539-2545.

$\mathrm{Au}$, W. W. L. (1993). The Sonar of Dolphins (Springer-Verlag, New York), p. 277.

Au, W. W. L. (2015). "History of dolphin biosonar research," Acoust. Today 11, 10-17.

Au, W. W. L., Houser, D. S., Finneran, J. J., Lee, W., Talmadge, L. A., and Moore, P. W. (2010). "The acoustic field on the forehead of echolocating Atlantic bottlenose dolphins (Tursiops truncatus)," J. Acoust. Soc. Am. 128(3), 1426-1433.

Au, W. W. L., Kastelein, R. A., Rippe, T., and Schooneman, N. M. (1999). "Transmission beam pattern and echolocation signals of a harbor porpoise (Phocoena phocoena)," J. Acoust. Soc. Am. 106(6), 3699-3705.

Au, W. W. L., Moore, P. W. B., and Pawloski, D. A. (1986). "Echolocation transmitting beam of the Atlantic bottlenose dolphin," J. Acoust. Soc. Am. 80, 688-691.

Au, W. W. L., and Simmons, J. A. (2007). "Echolocation in dolphins and bats," Phys. Today 60(9), 40-45.

Bérenger, J. P. (1994). "A perfectly matched layer for the absorption of electromagnetic waves," J. Comput. Phys. 114(2), 185-200.

Cranford, T. W. (1988). "Anatomy of acoustic structures in the spinner dolphin forehead as shown by X-ray computed tomography and computer graphics," in Animal Sonar: Processes and Performance, edited by P. E. Nachtigall and P. W. B. Moore (Plenum Publishing Co., New York), pp. 67-77.

Cranford, T. W. (1992). "Functional morphology of the odontocete forehead: Implications for sound generation," Ph.D. thesis, The University of California, Santa Cruz, CA.

Cranford, T. W. (2000). "In search of impulse sound sources in odontocetes," in Hearing by Whales and Dolphins, edited by W. W. L. $\mathrm{Au}$, A. N. Popper, and R. R. Fay (Springer-Verlag, New York), pp. $109-156$.

Cranford, T. W., Amundin, M., and Norris, K. S. (1996). "Functional morphology and homology in the odontocete nasal complex: Implications for sound Generation," J. Morphol. 228(3), 223-285.

Cranford, T. W., Trijoulet, V., Smith, C. R., and Krysl, P. (2014). "Validation of a vibroacoustic finite element model using bottlenose dolphin simulations: The dolphin biosonar beam is focused in stages," Bioacoustics 23(2), 161-194.
Finneran, J. J., Branstetter, B. K., Houser, D. S., Moore, P. W., Mulsow, J., Martin, C., and Perisho, S. (2014). "High-resolution measurement of a bottlenose dolphin's (Tursiops truncatus) biosonar transmission beam pattern in the horizontal plane," J. Acoust. Soc. Am. 136(4), 2025-2038.

Finneran, J. J., Mulsow, J., Branstetter, B. K., Moore, P. W., and Houser, D. S. (2016). "Nearfield and farfield measurements of dolphin echolocation beam patterns: No evidence of focusing," J. Acoust. Soc. Am. 140(2), $1346-1360$.

Harper, C. J., Mclellan, W. A., Rommel, S. A., Gay, D. M., Dillaman, R., and Pabst D. A. (2008). "Morphology of the melon and its tendinous connections to the facial muscles in bottlenose dolphins (Tursiops truncatus)," J. Morphol. 269(7), 820-839.

Houser, D. S., Finneran, J. J., Carder, D. A., Van Bonn, W., Smith, C., Hoh, C., Mattrey, R., and Ridgway, S. H. (2004). "Structural and functional imaging of bottlenose dolphin (Tursiops truncatus) cranial anatomy," J. Exp. Biol. 207(21), 3657-3665.

Jing, X., Xiao, Y., and Jing, R. (1982). "The acoustic function of the melon of Chinese River Dolphin (Lipotes vexillifer)," Acta Acust. 7(1), 14-22.

Kloepper, L., Buck, J. R., Smith, A. B., Supin, A. Y., Gaudette, J. E., and Nachtigall, P. E. (2015). "Support for the beam focusing hypothesis in the false killer whale," J. Exp. Biol. 218(15), 2455-2462.

Kloepper, L., Nachtigall, P. E., Donahue, M. J., and Breese, M. (2012). "Active echolocation beam focusing in the false killer whale, Pseudorca crassidens," J. Exp. Biol. 215(8), 1306-1312.

Krysl, P., Cranford, T. W., Wiggins, S. M., and Hildebrand, J. A. (2006). "Simulating the effect of high-intensity sound on cetaceans: Modeling approach and a case study for Cuvier's beaked whale (Ziphius cavirostris)," J. Acoust. Soc. Am. 120(4), 2328-2339.

Madsen, P. T., Lammers, M., Winsniewaska, D., and Beedholm, K. (2013). "Nasal sound production in echolocating delphinids (Tursiops truncates and Pseudorca crassidens) is dynamic, but unilateral: Clicking on the right side and whistling on the left side," J. Exp. Biol. 216(21), 4091-4102.

Madsen, P. T., Wisniewska, D., and Beedholm, K. (2010). "Single source sound production and dynamic beam formation in echolocating harbour porpoises (Phocoena phocoena)," J. Exp. Biol. 213(18), 3105-3110.

McKenna, M. F. (2005). "Comparative morphology of the odontocete melon: Functional and evolutionary interpretations," MSc thesis, San Diego State University, San Diego, CA.

Moore, P. W., Dankiewicz, L. A., and Houser, D. S. (2008). "Beamwidth control and angular target detection in an echolocating bottlenose dolphin (Tursiops truncatus)," J. Acoust. Soc. Am. 124(5), 3324-3332.

Norris, K. S., and Harvey, G. W. (1974). "Sound transmission in the porpoise head," J. Acoust. Soc. Am. 56(2), 659-664.

Norris, K. S., Prescott, J. H., Asa-Drian, P. V., and Perkins, P. (1961). "An experimental demonstration of echolocation behavior in the porpoise, Tursiops truncatus (Montagu)," Biol. Bull. 120(2), 163-176.

Soldevilla, M. S., McKenna, M. F., Wiggins, S. M., Shadwick, R. E., Cranford, T. W., and Hildebrand, J. A. (2005). "Cuvier's beaked whale (Ziphius cavirostris) head tissues: Physical properties and CT imaging," J. Exp. Biol. 208(12), 2319-2332.

Song, Z., Zhang, Y., Wei, C., and Wang, X. (2016). "Inducing rostrum interfacial waves by fluid-solid coupling in a Chinese river dolphin (Lipotes vexillifer)," Phys. Rev. E 93, 012411.

Wei, C., Au, W. W. L., Ketten, R. D., Song, Z., and Zhang, Y. (2017). "Biosonar signal propagation in the harbor porpoise's (Phocoena phocoena) head: The role of various structures in the formation of the vertical beam," J. Acoust. Soc. Am. 141(6), 4179-4187.

Wei, C., Au, W. W. L., Song, Z., and Yu, Z. (2016). "The role of various structures in the head on the formation of the biosonar beam of the baiji (Lipotes vexillifer)," J. Acoust. Soc. Am. 139(2), 875-880.

Wei, C., Song, Z., Au, W. W. L., Zhang, Y., and Wang, D. (2018). "A numerical evidence of biosonar beam forming of a neonate Yangtze finless porpoise (Neophocaena asiaeorientalis)," J. Comput. Acoust. 26, 1850009.

Wei, C., Wang, Z., Song, Z., Wang, K., Wang, D., Au, W. W. L., and Yu, Z. (2015). "Acoustic property reconstruction of a neonate Yangtze finless porpoise's (Neophocaena asiaeorientalis) head based on CT Imaging," PLoS One 10(4), e0121442.

Wei, C., Yu, Z., and Au, W. W. L. (2014). "Simulation of ultrasound beam formation of baiji (Lipotes vexillifer) with a finite element model," J. Acoust. Soc. Am. 136(1), 423-429.

Zhang, Y., Song, Z., Wang, X., Cao, W., and Au, W. W. L. (2017). "Directional acoustic wave manipulation by a porpoise via multiphase forehead structure," Phys. Rev. Appl. 8, 064002. 